\title{
The effect of predation on the prevalence and aggregation of pathogens in prey
}

\author{
Min $\mathrm{Su}^{\mathrm{a}, *}$, Cang Hui ${ }^{\mathrm{b}, * *}$ \\ a School of Mathematics, Hefei University of Technology, Hefei 230009, China \\ ${ }^{\mathrm{b}}$ Centre for Invasion Biology, Department of Botany and Zoology, University of Stellenbosch, Private Bag X1, Matieland 7602, South Africa
}

\section{A R T I C L E I N F O}

\section{Article history}

Received 20 December 2010

Received in revised form 9 May 2011

Accepted 26 May 2011

\section{Keywords:}

Eco-epidemiology

Predation pressure

Spatial structure

Join-count statistics

Pair approximation

\begin{abstract}
A B S T R A C T
Although pathogens and predators have been widely used as bio-control agents against problematic prey species, little has been done to examine the prevalence and aggregation of pathogens in spatially structured eco-epidemiological systems. Here, we present a spatial model of a predator-prey/host-parasite system based on pair approximation and spatially stochastic simulations, with the predation pressure indicated by predator abundance and predation rates. Susceptible prey can not only be infected by contacting adjacent infected individuals but also by the global transmission of pathogens. The disease prevalence was found to follow a hump-shaped function in response to predation pressure. Moreover, predation pressure was not always negatively correlated with pathogen aggregation as proposed from empirical studies, but depending on the level of predation pressure. Highly connected site network facilitated the parasites infection, especially under high predation pressure. However, the connectivity of site network had no effect on the prevalence and aggregation of pathogens that can infect health prey through global transmission. It is thus possible to better design biological control strategies for target species by manipulating predation pressure and the range of pathogen transmission.
\end{abstract}

(c) 2011 Elsevier Ireland Ltd. All rights reserved.

\section{Introduction}

Pathogen infection and predation are distinctive but analogous inter-specific interactions, with each an important field of research in its own right (Bairagi et al., 2007; Raffel et al., 2008; Mata-Machuca et al., 2010; Kihara et al., 2011). Eco-epidemiology that considers both ecological and epidemiological dynamics ties these two fields together and has attracted increasing attention (Chattopadhyay and Bariagi, 2001; Webb et al., 2007a; Bairagi et al., 2007; Su et al., 2009a; Greenman and Hoyle, 2010). Studies in ecoepidemiology have provided increasing insights to the complex dynamics in the system and their applications in conservation management, such as the biological control of problematic species using their natural enemies through the interplay of disease transmission and predation (Holt and Roy, 2007; Greenman and Hoyle, 2010). For instance, although predation has been viewed as an important force to prevent successful invasion of pathogens into prey (Packer et al., 2003; Bairagi et al., 2007), the prevalence of pathogenic diseases can, nonetheless, enhance the predation risk (Hethcote et al., 2004; Hatcher et al., 2006; Williams, 2008). As such, understanding such complex processes and dynamics in eco-epidemiological systems and elucidating the impact of predation on the control of epidemics are necessary and have important implications in wildlife conservation and management.

\footnotetext{
* Corresponding author. Tel.: +86 551 2902592; fax: +86 5512902500

** Corresponding author.

E-mail addresses: sum04@163.com (M. Su), chui@sun.ac.za (C. Hui).
}

Theoretical studies have provided certain propositions regarding the impacts of predation on the pathogen loads in natural predator-prey/host-parasitoid systems (Packer et al., 2003; Bairagi et al., 2007; Roy and Holt, 2008; Williams, 2008; Greenman and Hoyle, 2010). Evidently, because predators prefer infected prey as easy targets, they can potentially alter the prevalence of disease in prey population (Hudson et al., 1992; Packer et al., 2003; Ostfeld and Holt, 2004; Hall et al., 2005; Roy and Holt, 2008). Packer et al. (2003) thus suggest that the removal of predators can be indirectly detrimental to prey and facilitate pathogen invasion and transmission (also see Bairagi et al., 2007; Williams, 2008). However, recent work has questioned the generality of Packer et al.'s proposition by demonstrating results that depend on prey's mechanisms of population regulation (Holt and Roy, 2007; Roy and Holt, 2008). For instance, if considering the acquired immunity in prey, the overall relationship between pathogen prevalence and predator abundance could be hump-shaped (Holt and Roy, 2007). Enhanced predation pressure (either by manipulating predator density or enhancing predation efficiency) could also facilitate the transmission of a pathogen under certain circumstances (Greenman and Hoyle, 2010).

Preference in predation can also have a bearing on pathogen transmission. Although studies often consider that predators prefer infected (less active) prey (Chattopadhyay and Bariagi, 2001; Hethcote et al., 2004; Bairagi et al., 2007), it has also been suggested that such preferential predation could also depend on the type of pathogens and the life-history characteristics of predators (Dawkins, 1982; Bhattacharyya and Mukhopadhyay, 2010). 
The effect of predation preference on pathogen transmission and prevalence is worthy of additional investigation.

Another important factor in eco-epidemiology is the spatial structure of prey which affects and is also affected by pathogen transmission (Bonsall and Hassell, 2000; Su et al., 2009a). For a parasitic infection that transmits to nearby hosts via direct contact, traditional analyses based on the mean-field assumption that individuals are well mixed and have equal probability to encounter one another could be inappropriate. In this regard, using pair approximation to consider the spatially explicit dynamics has allowed for better understanding the role of spatial structure (autocorrelation) in a wide range of ecological and epidemiological questions (e.g. Satō et al., 1994; Tilman and Kareiva, 1997; Hui and McGeoch, 2007; Webb et al., 2007a,b; Okuyama, 2008). Invasion and persistence of pathogens have been shown to become more difficult under realistic spatial structure as opposed to under the mean-field assumption (Boots and Sasaki, 1999; Bauch, 2005; Webb et al., 2007a). Various degrees of spatial autocorrelation have been observed in healthy and infected prey (Boots and Sasaki, 1999), and it is therefore necessary to examine the effect of a full range of spatial autocorrelation on the eco-epidemiological dynamics.

One important finding when considering the spatial structure in eco-epidemiology is the role of aggregated distribution on the persistence of prey and pathogens (Shaw et al., 1998; Joly and Messier, 2004). Recent works further suggest that the aggregation of pathogen distribution can be sensitive to predation (Rousset, 1996; Joly and Messier, 2004). For instance, Joly and Messier (2004) show that the aggregation of parasites (Echinococcus granulosus) in the moose hosts could be reduced by the parasite-induced vulnerability to predation by wolfs. Clearly, it is impossible to quantify the aggregation of parasites and pathogens using the mean-field approximation (Su et al., 2009b). Pair approximation enables such spatially explicit analysis of species aggregated distribution (Satō et al., 1994; Su et al., 2009b) and is thus suitable for examining the potential effect of the aggregation in eco-epidemiological systems.

We here explore the effect of predation (pressure and preference) on the epidemic transmission in spatially structured prey populations. Using a combination of approximation techniques and spatially stochastic simulations, we examine the effect of adding spatial components on the pathogen transmission and aggregation in an eco-epidemiological system. Specifically, we examine how local reproduction and a mixture of local and global pathogen transmission on the prevalence and aggregation of diseases in prey. We provide a threshold for pathogen invasion based on the analysis of invasion matrix (Greenman and Hoyle, 2010). Given the conceptual similarity between the join-count statistics and pair approximation (Hui et al., 2006, 2010; Su et al., 2009b), we further explore the aggregation degree of infected prey in response to different predation pressure and preference, as well as different transmission types, which has not been previously studied for ecoepidemiological systems. These analyses enable us to address the following questions: (1) how does the mean-field dynamics change after considering the spatial structure of prey and diseases? (2) How do the predation pressure and preference of predators affect the invasion, prevalence and aggregation of pathogens? (3) How does the spatial structure of infected prey change in response to different levels of predation pressure and transmission rates? Answers to these questions can shed light on the entangled dynamics and processes in eco-epidemiological systems and provide clues for more efficient biodiversity conservation and management.

\section{Model}

Pair approximation is a method for analyzing the first-order join-count spatial autocorrelation (Fortin and Dale, 2005; Hui et al., (a)
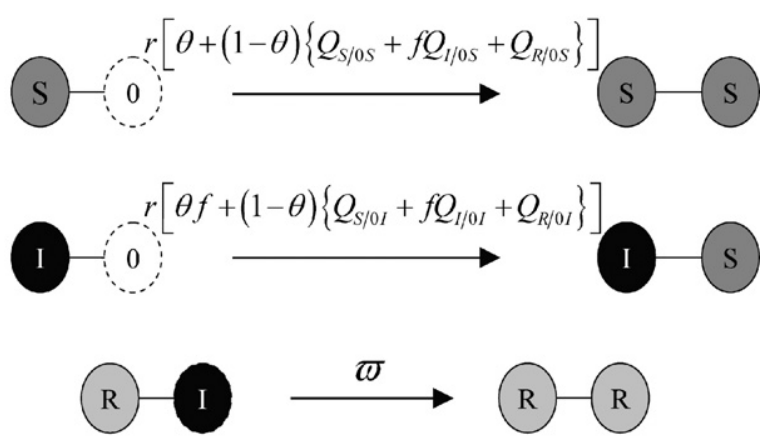

(b)

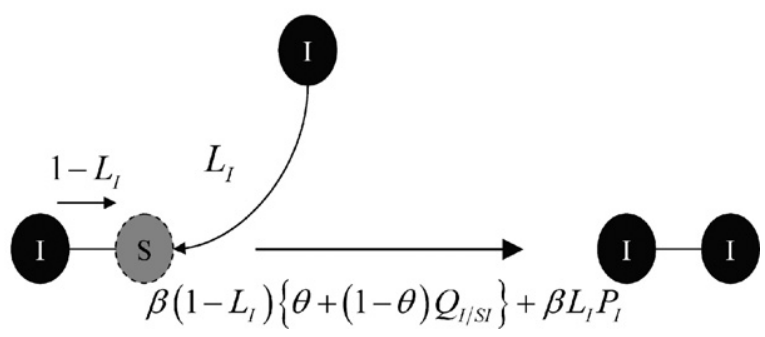

Fig. 1. (a) The reproductive and recover process with possible pair states and their transition probability. (b) The infection process of a susceptible individual who can be infected from a nearest-neighbor site at probability $1-L_{I}$ and globally from a distant site at probability $L_{I}$.

2010) and for tracing the global and local densities in a dynamical system (Keeling and Rand, 1996; Hiebeler, 2005; Su et al., 2009b). We designed a pair approximation model for a predator-prey system, where the prey is also the host of a transmissible pathogen in an epidemiological system and can either be susceptible to, infected by, or recovered from the pathogenic disease. Following Packer et al. (2003) and Roy and Holt (2008), we only considered the predator abundance $\left(P_{C}\right)$ as a surrogate for predation pressure. The model was run on a network of sites, with each representing either empty (0) or occupied by a susceptible prey $(S)$, an infected one $(I)$ or a recovered individual $(R)$. Each susceptible or recovered individual reproduced at a rate of $r$ and deposited its offspring into adjacent empty sites (Fig. 1a). Reproduction from infected individuals was also included but at a lower rate $(r f$, where $f \in[0,1])$. Susceptible prey can be infected by the pathogen either through global transmission from any other infected prey (at a rate of $\beta L_{I}$, where $\beta$ indicates the transmission rate of infection and $L_{I}(0 \leq$ $L_{I} \leq 1$ ) denotes the proportion of global transmission) or through direct contact with surrounding infected individuals (at a rate of $\beta\left(1-L_{I}\right)$, Fig. $\left.1 \mathrm{~b}\right)$. The recovery from infection (at a rate of $\varpi$ ) can lead to immunity from further infection (Fig. 1a). The mortality of hosts (prey) consisted of three components: intrinsic death rate $\left(m_{\sigma 0}, \sigma \in\{S, I, R\}\right)$, pathogen-induced death rate ( $\mu$ and predationinduced death rate $\alpha_{\sigma} P_{C}$, where $\alpha_{\sigma}$ is the predation rate). The enhanced mortality due to predation can be represented as $m_{\sigma}=$ $m_{\sigma 0}+\alpha_{\sigma} P_{C}$, where we assumed $m_{S 0}=m_{R 0}=m$ for susceptible and recovered prey and $m_{I 0}=m+\mu$ for infected prey. The definition of parameters and estimated values are summarized in Table 1.

Let the global density $P_{\sigma^{\prime}}(t)\left(\sigma^{\prime} \in\{S, I, R, 0\}\right)$ denote the probability that a randomly chosen site is in state $\sigma^{\prime}$ at time $t$. Doublet density $P_{\sigma^{\prime} \sigma^{\prime \prime}}$ represents the probability that a randomly chosen pair of two adjacent sites is in state $\sigma^{\prime} \sigma^{\prime \prime}$. Local density $Q_{\sigma^{\prime} / \sigma^{\prime \prime}}$ represents the conditional probability that a randomly chosen neighbor of a site in state $\sigma^{\prime \prime}$ is in state $\sigma^{\prime}$, and $Q_{\sigma^{\prime} / \sigma^{\prime \prime} \sigma^{\prime \prime \prime}}$ is the conditional probability that a randomly chosen neighbor of the $\sigma^{\prime \prime}$ site in a $\sigma^{\prime \prime} \sigma^{\prime \prime \prime}$ pair is in state $\sigma^{\prime}\left(\sigma^{\prime \prime}, \sigma^{\prime \prime \prime} \in\{S, I, R, 0\}\right)$. By comparing the global and local densities of prey, we can classify the spatial distribution of individuals into aggregated, segregated and random (Fortin and Dale, 2005; Hui et al., 2006, 2010). A spatially aggregated 
Table 1

Definition of the parameters used in the model.

\begin{tabular}{|c|c|c|}
\hline Parameter & Definition & Default value \\
\hline$r$ & Reproduction rate of susceptible and recovered & 1 \\
\hline$f$ & Fractional rate of infected, $f \in[0,1]$ & 0.8 \\
\hline$\beta$ & Transmission rate & - \\
\hline$L_{I}$ & Proportion of global transmission, $L_{I} \in[0,1]$ & $0,0.5$ or 1 \\
\hline$\varpi$ & Recovery rate & 0.1 \\
\hline$P_{C}$ & Predator abundance & - \\
\hline$m$ & Intrinsic mortality rate of prey & 0.01 \\
\hline$\mu$ & Pathogen-induced death rate & 0.01 \\
\hline$\alpha_{S}$ & Predation rate on susceptible prey, then $m_{S}=m+\alpha_{S} P_{C}$ is the enhanced mortality rate due to predation & - \\
\hline$\alpha_{I}$ & Predation rate on infected prey, then $m_{I}=m+\mu+\alpha_{I} P_{C}$ is the mortality rate due to infection and predation & - \\
\hline$\alpha_{R}$ & Predation rate on recovered prey, then $m_{R}=m+\alpha_{R} P_{C}$ is the enhanced mortality rate of recovered & - \\
\hline$z$ & Neighbor size & 4 or 8 \\
\hline
\end{tabular}

distribution implies a positive first-order spatial autocorrelation between the states of two adjacent sites (i.e. a positive clumping degree: $C_{\sigma^{\prime}}=Q_{\sigma^{\prime} / \sigma^{\prime}}-P_{\sigma^{\prime}}>0$, where $\sigma^{\prime} \in\{S, I, R, 0\}$ ). Analogously, a spatially random distribution implies $C_{\sigma^{\prime}}=0$, and a spatially segregated distribution has $C_{\sigma^{\prime}}<0$ (Hui and McGeoch, 2007).

The pair approximation model of the above described ecoepidemiological system then yields the following differential equations:

$$
\begin{aligned}
& P_{S}^{\prime}=r P_{0}\left(Q_{S / 0}+f Q_{I / 0}+Q_{R / 0}\right)-m_{S} P_{S}-\beta P_{S}\left[\left(1-L_{I}\right) Q_{I / S}+L_{I} P_{I}\right] \\
& P_{I}^{\prime}=\beta P_{S}\left[\left(1-L_{I}\right) Q_{I / S}+L_{I} P_{I}\right]-\left(m_{I}+\varpi\right) P_{I} \\
& P_{R}^{\prime}=\varpi P_{I}-m_{R} P_{R} \\
& P_{0}^{\prime}=-r P_{0}\left(Q_{S / 0}+f Q_{I / 0}+Q_{R / 0}\right)+m_{S} P_{S}+m_{I} P_{I}+m_{R} P_{R} \\
& P_{S S}^{\prime}=2 r\left[\theta+(1-\theta)\left\{Q_{S / 0 S}+f Q_{I / 0 S}+Q_{R / 0 S}\right\}\right] P_{S O}-2 m_{S} P_{S S} \\
& -2 \beta P_{S S}\left\{\left(1-L_{I}\right)(1-\theta) Q_{I / S S}+L_{I} P_{I}\right\} \\
& P_{I I}^{\prime}=2 \beta P_{I S}\left(1-L_{I}\right)\left\{\theta+(1-\theta) Q_{I / S I}\right\}+2 \beta L_{I} P_{I} P_{I S}-2\left(m_{I}+\varpi\right) P_{I I} \\
& P_{R R}^{\prime}=2 \varpi P_{I R}-2 m_{R} P_{R R} \\
& P_{00}^{\prime}=-2 r(1-\theta) P_{00}\left(Q_{S / 00}+f Q_{/ / 00}+Q_{R / 00}\right) \\
& +2 m_{S} P_{S O}+2 m_{I} P_{I 0}+2 m_{R} P_{R O} \\
& P_{S 0}^{\prime}=r P_{00}(1-\theta)\left\{Q_{S / 00}+f Q_{I / 00}+Q_{R / 100}\right\} \\
& -r P_{S 0}\left[\theta+(1-\theta)\left\{Q_{S / 0 S}+f Q_{I / O S}+Q_{R / 0 S}\right\}\right] \\
& -m_{S} P_{S 0}+m_{S} P_{S S}+m_{R} P_{R S}+m_{I} P_{I S} \\
& -\beta P_{S 0}\left\{\left(1-L_{I}\right)(1-\theta) Q_{I / S 0}+L_{I} P_{I}\right\} \\
& P_{I 0}^{\prime}=\beta P_{S 0}\left\{\left(1-L_{I}\right)(1-\theta) Q_{I / S 0}+L_{I} P_{I}\right\}+m_{S} P_{I S}+m_{I} P_{I I}+m_{R} P_{I R} \\
& -\left(m_{I}+\varpi\right) P_{I 0}-r P_{I 0}\left[\theta f+(1-\theta)\left\{Q_{S / 0 I}+f Q_{I / 0 I}+Q_{R / 0 I}\right\}\right] \\
& P_{R 0}^{\prime}=\varpi P_{I 0}+m_{S} P_{R S}+m_{I} P_{R I}+m_{R} P_{R R}-m_{R} P_{R 0} \\
& -r P_{R 0}\left[\theta+(1-\theta)\left\{Q_{S / 0 R}+f Q_{I / 0 R}+Q_{R / 0 R}\right\}\right] \\
& P_{R S}^{\prime}=r P_{R 0}\left[\theta+(1-\theta)\left\{Q_{S / 0 R}+f Q_{I / 0 R}+Q_{R / 0 R}\right\}\right]-\left(m_{S}+m_{R}\right) P_{R S} \\
& +\varpi P_{I S}-\beta P_{R S}\left\{\left(1-L_{I}\right)(1-\theta) Q_{I / S R}+L_{I} P_{I}\right\} \\
& P_{I S}^{\prime}=r P_{I 0}\left[\theta f+(1-\theta)\left\{Q_{S / 0 I}+f Q_{I / 0 I}+Q_{R / 0 I}\right\}\right] \\
& -\beta P_{S S}\left\{\left(1-L_{I}\right)(1-\theta) Q_{I / S S}+L_{I} P_{I}\right\} \\
& -\beta P_{I S}\left[\left(1-L_{I}\right)\left\{\theta+(1-\theta) Q_{I / S I}\right\}+L_{I} P_{I}\right] \\
& -\left(m_{S}+m_{I}+\varpi\right) P_{I S} \\
& P_{I R}^{\prime}=\beta P_{R S}\left\{\left(1-L_{I}\right)(1-\theta) Q_{I / S R}+L_{I} P_{I}\right\}+\varpi P_{I I} \\
& -\left(m_{I}+m_{R}+\varpi\right) P_{I R}
\end{aligned}
$$

where $\theta=1 / z$ ( $z$ is the number of adjacent sites in the network). In the above equations, the following equalities from probability rules hold: $P_{\sigma^{\prime} \sigma^{\prime \prime}}=P_{\sigma^{\prime}} Q_{\sigma^{\prime \prime} / \sigma^{\prime}}=P_{\sigma^{\prime \prime} \sigma^{\prime}}=P_{\sigma^{\prime \prime}} Q_{\sigma^{\prime} / \sigma^{\prime \prime}}, P_{\sigma^{\prime}}=$ $\sum_{\sigma^{\prime \prime}} P_{\sigma^{\prime} \sigma^{\prime \prime}}, \sum_{\sigma^{\prime \prime}} Q_{\sigma^{\prime \prime} / \sigma^{\prime}}=1$ and $P_{0}+P_{S}+P_{I}+P_{R}=1$. According to the pair approximation (i.e. $Q_{\sigma^{\prime} / \sigma^{\prime \prime} \sigma^{\prime \prime \prime}} \approx Q_{\sigma^{\prime} / \sigma^{\prime \prime}}$ ) (Iwasa, 2000; Su et al., 2009b), there are only nine independent variables in above equations, namely $P_{S S}, P_{I I}, P_{R R}, P_{S 0}, P_{I 0}, P_{R 0}, P_{I S}, P_{I R}$ and $P_{R S}$. All other singlet and doublet probabilities can be calculated from these nine variables. For comparison, we also presented the results under the mean-field assumption (i.e. $\left(Q_{\sigma^{\prime} / \sigma^{\prime \prime}}=P_{\sigma^{\prime}}\right)$.

Given the complexity of above equations, we used a method of invasion analysis to explore the dynamic behaviors of this ecoepidemiological system. This method analyzes the invasion matrix when the pathogen prevalence is extremely low and thus enables to estimate the threshold condition for pathogen invasion (Greenman and Hoyle, 2010). The invasion analysis further assumes that both the abundance of infected and recovered host populations is low and can be ignored when calculating the equilibrium of resident (susceptible) hosts (Okuyama, 2008; Su et al., 2009; Greenman and Hoyle, 2010). Invasiveness of a pathogen can then be defined as whether a small number of initially infected hosts can increase or not when the resident hosts are at their equilibrium (Iwasa, 2000; Okuyama, 2008; see full details in Appendix A).

To validate our analysis, a spatial stochastic simulation (cellular automaton) was also built on a two-dimensional lattice network $(200 \times 200$ sites $)$. Synchronized updating and periodic boundaries were used (Hui and McGeoch, 2007; Su et al., 2009a). Each simulation was run for 400 generations, where the system achieved equilibrium. Initially, host individuals (susceptible, infected and recovered) were randomly located among sites because results of such models are not sensitive to initial conditions (Webb et al., 2007a; Su et al., 2009b). Different network connectivity ( $z=4$ or 8), predation rate of infected hosts $\left(\alpha_{I}=0.2\right.$ or 0.5$)$ and proportion of global transmission $\left(L_{I}=0\right.$ or 0.5$)$ were examined. Taken together, the numerical solution of the pair approximation model, the invasion analysis and the spatial stochastic simulation enabled us to examine how transmission rate $(\beta)$, predation pressure (including predation rate, $\alpha_{\sigma}$, and predator abundance, $\left.P_{C}\right)$, the proportion of global transmission $(L)$ and the number of neighboring sites $(z)$ affect the invasion threshold, prevalence of disease $\left(\rho^{*}\right)$ and the clumping (aggregation) degree of infected prey $\left(C_{I}^{*}\right)$ in this ecoepidemiological system.

\section{Results}

The invasion threshold $\left(\beta_{c}\right.$, the minimum transmission rate for pathogen to successfully invade the host population) for both the pair and mean-field approximations was examined as a function of predation pressure consisting of two components: indiscriminate predation (i.e. $\alpha_{S}=\alpha_{I}=\alpha_{R}$, denoted by $\alpha$ ) and predator abundance $\left(P_{C}\right)$ (Fig. 2). The invasion threshold increased monotonically with 


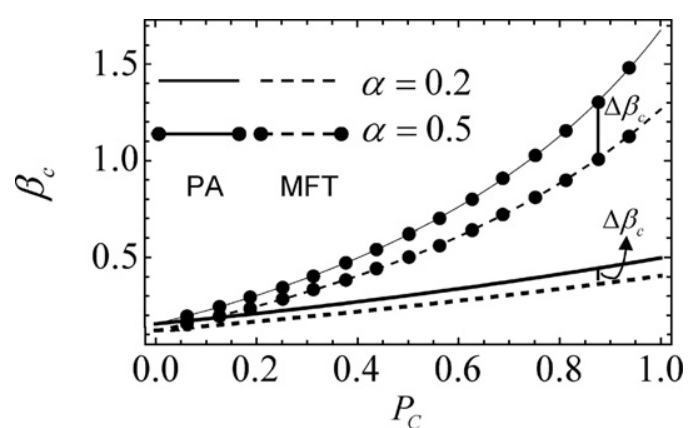

Fig. 2. Invasion threshold as a function of predator abundance based on two different approximation models with various predation rates. 'MFT' and 'PA' represent the mean-field approximation and pair approximation models, respectively. $\alpha$ indicates indiscriminate predation rate, i.e., $\alpha_{S}=\alpha_{I}=\alpha_{R}=\alpha$. Other parameters include: $r=1, f=0.8, \varpi=0.1, m=0.01, \mu=0.01, L_{I}=0, z=8$.

predation pressure for both pair and mean-field approximations, suggesting that enhanced predation pressure could prevent the invasion of pathogens. The estimates of invasion threshold from pair approximation were higher than from the mean-field approximation (Fig. 2), suggesting that the easiness of pathogen invasion could be exaggerated by spatially implicit models. Furthermore, gaps between the invasion thresholds from the two approximations (indicated by $\Delta \beta_{c}$ in Fig. 2) widened with the increase of predator abundance $\left(P_{C}\right)$ and predation rates $(\alpha)$, emphasizing that it become more appropriate to use spatially explicit analyses when the predation pressure is high.

To further examine the impact of predation pressure on the epidemic dynamics, we plotted the prevalence of disease $\left(\rho^{*}\right)$ and the clumping (aggregation) degree of infected prey $\left(C_{I}^{*}\right)$ under different levels of predation pressure (Fig. 3). The prevalence of disease exhibited a nonlinear humped-shape with predation pressure (Fig. 3a). Notably, the indiscriminate predation rates $(\alpha)$ had a relatively similar effect as the predation abundance $\left(P_{C}\right)$ on the prevalence of disease $\left(\rho^{*}\right)$ (Fig. 3a). These results suggest that a moderate level of predation pressure can lead to a high prevalence of disease, whereas extreme levels of predation pressure (either extremely high or low) can expel the pathogens (or infected prey) from the population. The clumping degree of infected hosts $\left(C_{I}^{*}>0\right)$ also exhibited an intricate pattern with predation pressure (Fig. $3 \mathrm{~b})$, where the clumping degree $\left(C_{I}^{*}\right)$ declines with low predation pressure and was negatively correlated with the disease prevalence (comparing Fig. 3a and b).

While keeping the predation rates of susceptible and recovered prey constant $\left(\alpha_{S}=\alpha_{R}=0.2\right)$, changing predation rate of infected prey to represent that the predator completely avoid, indiscriminate or prefer infected prey $\left(\alpha_{I}=0,0.2,0.5\right.$, respectively) led to different levels of disease prevalence. With the increasing preference of infected prey, we observed a decline of disease prevalence

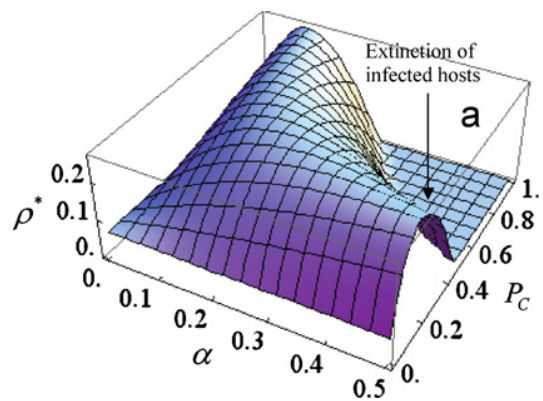

(Fig. 4a and b) but an increase of pathogen aggregation (Fig. 4c and d). If predator avoids foraging on infected prey $\left(\alpha_{I}=0\right)$, both the hump-shaped prevalence and concave shaped aggregation (as a function of predator abundance) disappeared, leading to a positive correlation between $\rho^{*}$ and $P_{C}$, and a negative correlation between $C_{I}^{*}$ and $P_{C}$ (Fig. 4). Furthermore, the prevalence and aggregation of disease were also affected by the proportion of global transmission $\left(L_{I}\right)$ and infection rate $(\beta)$ (Fig. 4). Evidently, low values of $L_{I}$ or $\beta$ resulted in a low prevalence of disease but high degree of aggregation, and the effects were also amplified under high predation pressure (Fig. 4).

Local infection in this eco-epidemic system can be characterized by the number of neighboring sites ( $z$; that is the spatial structure of the site network) and the proportion of global transmission $\left(L_{I}\right)$. First, both the pair approximation and stochastic simulation models showed that a highly connected site network (i.e. a high number of neighboring sites, $z$ ) can facilitate the local transmission of pathogens ( $z=4$ and $8 ; L_{I}=0$ ) (Fig. 5 ). This is because low connectivity reduces the chance of infected prey encountering healthy prey. Moreover, due to inherent stochasticity, the spatial stochastic simulation predicted a lower disease prevalence $\left(\rho^{*}\right)$ and earlier extinction of disease than pair approximation (Fig. 5). If the infection was only through global transmission $\left(L_{I}=1\right.$; note that the deposition of prey offspring was still between adjacent sites), the estimates of disease prevalence from the pair approximation model and the spatial stochastic simulation were almost similar to the prediction from the mean-field approximation, regardless of the spatial structure of the site network (Fig. 5). Both the mean-field approximation and completely global infection $\left(L_{I}=1\right)$ can lead to a zero clumping degree of infected prey (i.e. $C_{I}^{*}=0$ ). In contrast, low connectivity of the site network $(z=4)$ and completely local infection $\left(L_{I}=0\right)$ produced the highest degree of aggregation of infected prey (inset of Fig. 5). Clearly, the mode of transmission (local versus global) was the determinant of the spatial structure in this eco-epidemic system.

The spatial stochastic simulation supported above results (Fig. 6). With the increase of predator abundance $\left(P_{C}\right.$; comparing the left panel with the right panel in Fig. 6), the number of sites occupied by infected hosts declined. The increase of predation rate $\left(\alpha_{I}\right)$ also resulted in the decline of sites with infected prey (comparing Fig. 6a with c). The results further support that high predation pressure can impede the transmission of pathogen, consistent with predictions from the pair approximation model. Furthermore, also as expected from pair approximation, the proportion of global transmission, $L_{I}$ was found to favor the epidemic transmission from the stochastic simulation model (comparing with Fig. 6b and d).

\section{Discussion}

Eco-epidemiological interaction is common in ecosystems, where predation can greatly affect the pathogen invasion and

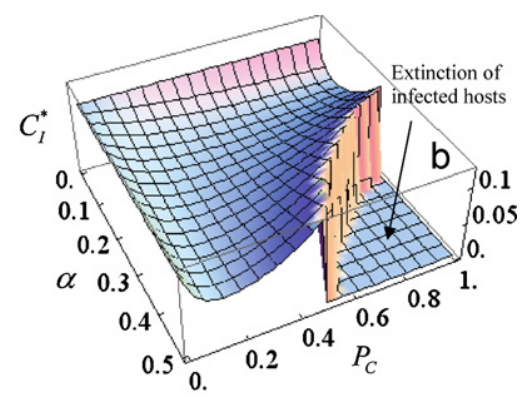

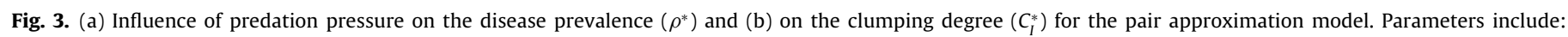
$r=1, f=0.8, \beta=0.6, \varpi=0.1, m=0.01, \mu=0.01, L_{I}=0, z=8$. 

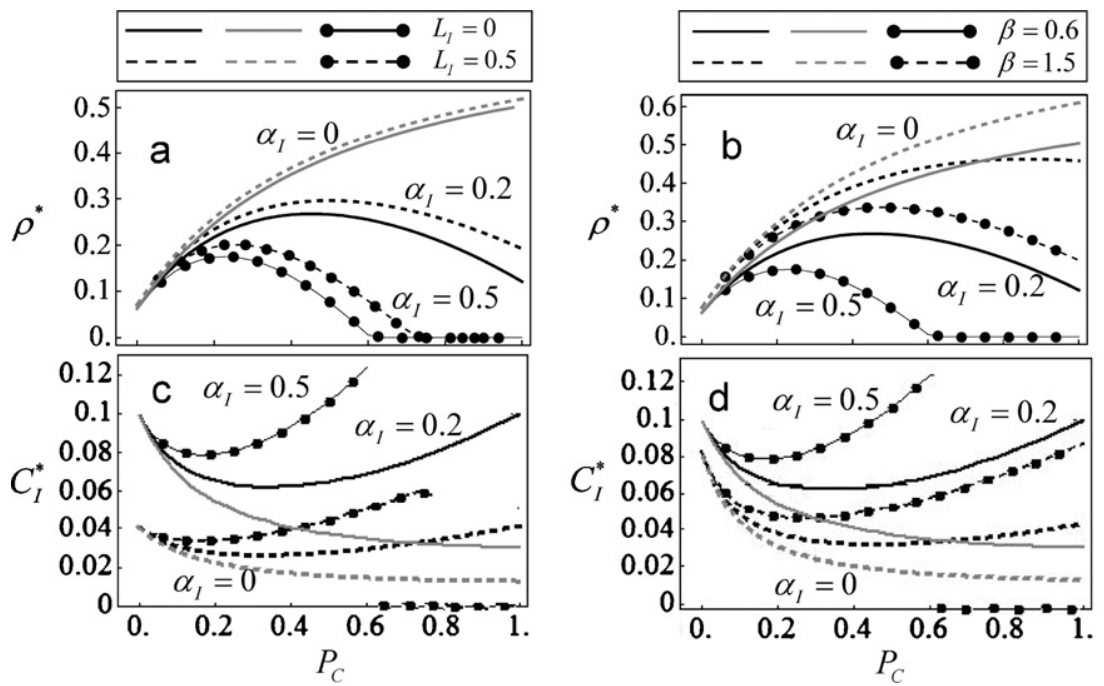

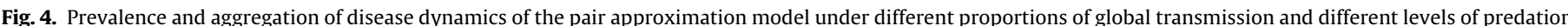

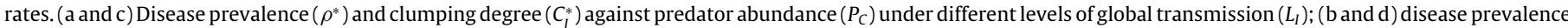

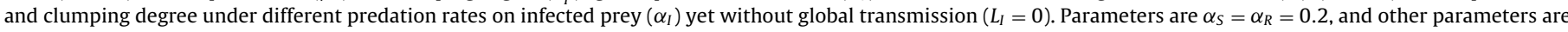
the same as in Fig. 3.

pathogen transmission (Holt and Roy, 2007; Bairagi et al., 2007; Roy and Holt, 2008; Su et al., 2009a; Greenman and Hoyle, 2010). Previous studies based on non-spatially structured SIR models have shown that predation not only can suppress the outbreak of pathogens in host populations but also facilitate infectious diseases, pending on the abundance of predators (Holt and Roy, 2007; Roy and Holt, 2008). Using pair approximation and stochastic simulation of a spatial SIR model with a mixture of local and global transmission, we here examined the synergistic effects of predation pressure, infection rate and the spatial structure of site network on the prevalence and aggregation of disease. Notably, our results confirmed that the prevalence of disease do exhibit a hump-shaped form in response to predation pressure, consistent with the proposition from Holt and Roy (2007). It is also evident that to maintain a high level of disease prevalence, there could exist a compensatory effect between the two components of predation pressure indiscriminant predation rate and predator abundance (Fig. 4), as suggested by Roy and Holt (2008).

Predation pressure can also interact with other biotic and abiotic factors to influence the dynamics of pathogens (Williams, 2008; Roy and Holt, 2008; Baek, 2009; Nie et al., 2009; Sun et al., 2010). For instance, here we showed that the effects of transmission mode (i.e. the proportion of global transmission, $\left.L_{I}\right)$, transmission rate $(\beta)$ and the spatial structure of site network $(z)$ on the prevalence of disease were amplified under high predation pressure. Such amplification could be due to that high predation pressure can quickly deplete infected hosts in surrounding sites and thus makes the persistence of disease rely more on processes such as global transmission, high infection rate and highly connected site networks. Consequently, our results imply that the effect of pathogens (as biological control agents) could be dampened when the hosts face high predation pressure, especially for pathogens that infect prey through predation (Hudson et al., 1992).

Aggregation of pathogens in hosts is common (Shaw et al., 1998 ) and could be caused by biotic interactions (e.g. predation, Joly and Messier, 2004) or environmental heterogeneity (Su et al., 2009a,b). Previous studies have showed that predation can dramatically affect the aggregation of pathogens (Rousset, 1996; Joly and Messier, 2004). Joly and Messier (2004) assumed that parasiteinduced vulnerability to predation is a positive function of infection intensity, thus the heavily infected individuals are removed highly with the increased parasite-induced mortality. Then, the reduc-

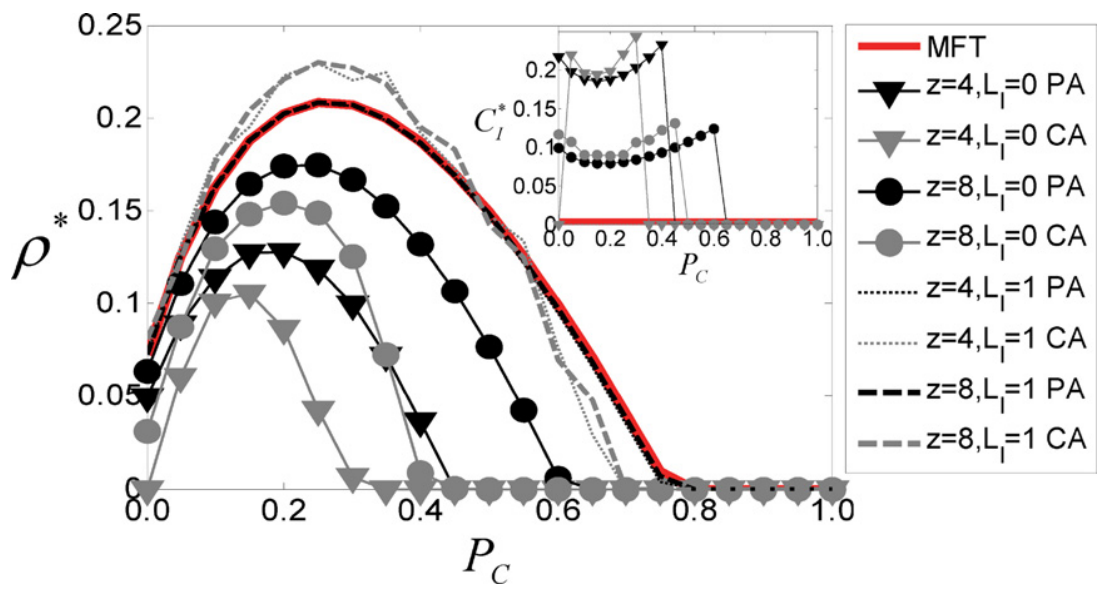

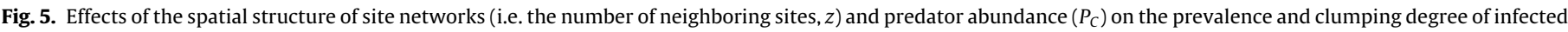

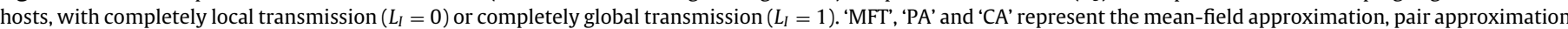
and cellular automata. Parameters are $\alpha_{S}=0.2, \alpha_{I}=0.5, \alpha_{R}=0.2$, and others are the same with Fig. 3. 


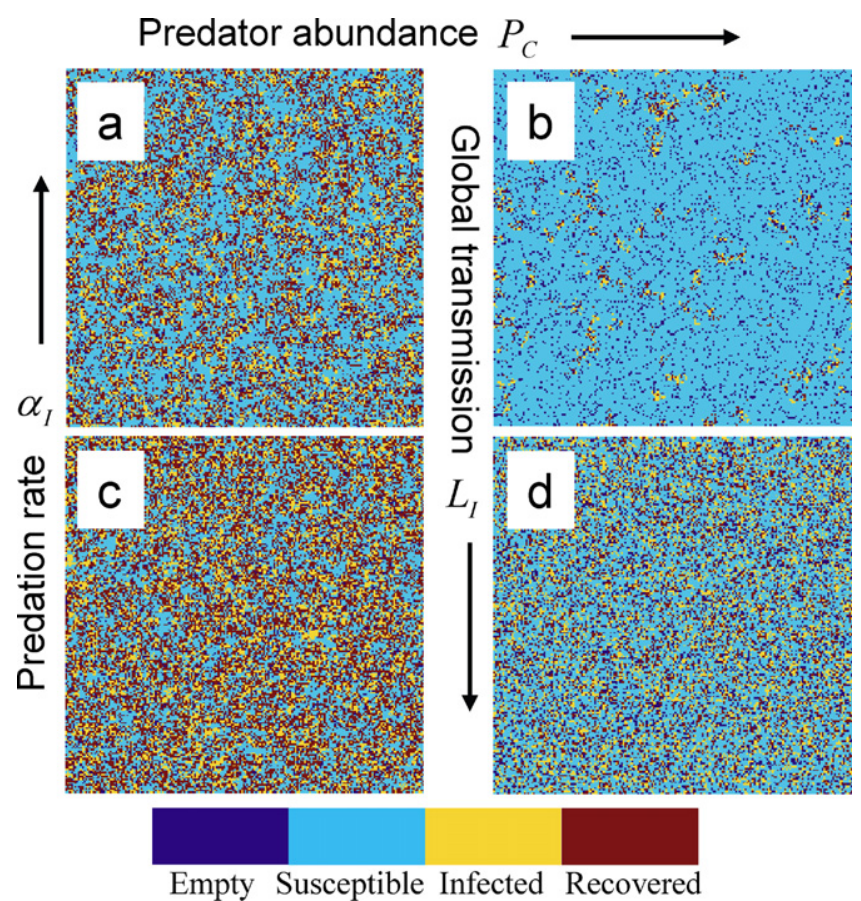

Fig. 6. Spatial patterns of prey from stochastic simulations after 400 time steps in $200 \times 200$ lattices. Parameters include: (left panel) $P_{C}=0.2, L_{I}=0, \alpha_{I}=0.5$ for (a) and $\alpha_{I}=0.2$ for (c); (right column) $P_{C}=0.4, \alpha_{I}=0.5, L_{I}=0$ for (b) and $L_{I}=0.5$ for (d). Other parameters include: $r=1, f=0.8, \beta=0.6, \alpha_{S}=0.2, \alpha_{R}=0.2, \varpi=$ $0.1, m=0.01, \mu=0.01, z=8$.

tion of infected hosts makes remaining parasites are distributed relatively evenly among the remaining hosts, which result in a negative correlation between predation pressure and pathogen aggregation (Joly and Messier, 2004). Our result revealed a concave relationship between predation pressure and the clumping degree of infected hosts, suggesting that Joly and Messier's proposition could be biased. Indeed, a dramatic increase of the aggregation levels of pathogens can also be expected under strong predation pressure (Woodcock et al., 2002; Su et al., 2009a).

Infectious pathogens can not only be transmitted by contacting surrounding individuals, but also be able to infect distant individuals via long-distance transmission. Previous studies have shown that poorly connected systems lead to a low level of disease prevalence and potential disease-driven extinction (Satō et al., 1994; Boots and Sasaki, 1999; Webb et al., 2007a; Su et al., 2009a). Although a mixture of local and global transmission has been proposed (Boots and Sasaki, 1999; Webb et al., 2007a), no previous research has revealed its impact on the prevalence of disease in site networks with different levels of connectivity. Our results elucidated the impact of transmission modes and clearly demonstrated that short-distance transmission can result in low prevalence and high aggregation of infection. Moreover, the nearer the infected hosts, the greater the risk of infection to susceptible hosts (O'Keefe, 2005). Then, accurate dispersal kernels (e.g. Gaussian kernel) for local transmission deserve further study. In our system, the alternative approach can be described as follows: for an infection site, the local transmission dispersing in $x$ and $y$ directions is described by a Gaussian dispersal kernel with mean zero $\left(\mu_{x}=\mu_{y}=0 ; \sigma_{x}=\sigma_{y}=\sigma\right)$, i.e., $k_{[x, y]}=\left(1 /\left(2 \pi \sigma^{2}\right)\right) \exp \left(-\left(x^{2}+\right.\right.$ $\left.\left.y^{2}\right) / 2 \sigma^{2}\right)$. Then, the transmission probability from a given infection source is weighted by $\left(k_{[x, y]}\right) / \sum_{x} \sum_{y} k$ (where $[x, y]$ is any site in the two-dimensional lattice network), which assuring that each probability summed to 1 (Slone, 2011).

One of the biggest challenges to further develop ecoepidemiology is to examine the interplay between disease infection and predation interactions in wild populations (Hatcher et al., 2006; Lafferty et al., 2008). Many empirical and theoretical works have investigated the pathogen-mediated effects on population and community dynamics (Rousset, 1996; Hatcher et al., 2006; Raffel, 2010). Theoretical studies have further shown that pathogen-induced modification of competitive and foraging abilities could affect the coexistence of multiple predator species in an intraguild predation system (Hatcher et al., 2006, 2008). We here considered three different predation preferences through changing the predation rates on infected hosts (avoiding, indiscriminant and preferring) and thus provided a broad picture of the interplay between disease infection and predation.

Moreover, controls that are varied in the parameters of the eco-epidemiological system can also be an important part of management's weaponry. For the invasion threshold, an analysis of its properties and sensitivity to parameter change can be carried out through algebraic formulas or numerical algorithms (Appendix A). According to the results, we can make consistent control strategies by changing the predation pressure. If the predator pressure cannot be directly controlled, one might be able to increase predator's survival rate or predation efficiency. However, further exploration on the dynamic control of disease in the eco-epidemiological system is still needed because of the complicated realities in this system (e.g. considering age structured and environmental heterogeneity). Considering these complexities in future studies can be important to derive optimal strategies for biological control and provide better conservation plans (Chattopadhyay et al., 2002; Delgado et al., 2006; Williams, 2008).

\section{Acknowledgements}

We are grateful to the editor and two anonymous reviewers for their constructive comments. This work was supported by the National Natural Science Foundation of China (31000192), China Postdoctoral Science Foundation (20100481159), the Fundamental Research Funds for the Central Universities (2010HGXJ0200) and the NRF Blue Skies Programme.

\section{Appendix A. Invasion Analysis of the Pair Approximation Model}

We calculated the critical transmissibility of disease in details. Let $\bar{v}$ denote the vector $\left(P_{I}, P_{I S}, P_{I I}, P_{I 0}, P_{I R}\right)$, and thus the equations involving infected prey $(I)$ can be written as:

$$
\frac{d \bar{v}}{d t}=M \cdot \bar{v}
$$

where
$M=\left(\begin{array}{ccc}-m_{I}-\varpi & \beta & 0 \\ 0 & \beta(1-\theta) Q_{S / S}-\beta\left\{\theta+(1-\theta) Q_{/ / S}\right\}-\left(m_{S}+m_{I}+\varpi\right) & 0 \\ 0 & 2 \beta\left\{\theta+(1-\theta) Q_{/ / S}\right\} & -2\left(m_{I}+\varpi\right) \\ 0 & \beta(1-\theta) Q_{0 / S}+m_{S} & m_{I} \\ 0 & \beta(1-\theta) Q_{R / s} & \varpi\end{array}\right.$ $\left.\begin{array}{cc}0 & 0 \\ r\left[\theta f+(1-\theta)\left\{Q_{S / 0}+Q_{R / 0}+f Q_{/ / 0}\right\}\right] & 0 \\ 0 & 0 \\ -\left(m_{I}+\varpi\right)-r\left[\theta f+(1-\theta)\left\{Q_{S / 0}+Q_{R / 0}+f Q_{/ / 0}\right\}\right] & m_{R} \\ 0 & -\left(m_{I}+m_{R}+\varpi\right)\end{array}\right)$ 
The global and local densities at their stationary values for the disease-free equilibrium can be derived as follows:

$Q_{S / S}=1-\frac{m_{S}}{r}, Q_{S / 0}=1-\frac{m_{S}}{r(1-\theta)}, Q_{0 / S}=\frac{m_{S}}{r}, Q_{0 / 0}=\frac{m_{S}}{r(1-\theta)}$,

$P_{S}=\frac{r(1-\theta)-m_{S}}{r(1-\theta)-\theta m_{S}}$ and $P_{0}=1-P_{S}$

and zero values for the remaining variables.

By substituting these above stationary values for the diseasefree equilibrium into the invasion matrix $(M)$, we can calculate the characteristic equation by evaluating the determinant $|M-\lambda I|$, which gives a characteristic polynomial:

$\left(\lambda+m_{I}+\varpi\right)^{2} H(\lambda)$,

where $H(\lambda)=\lambda^{4}+c_{3} \lambda^{3}+c_{2} \lambda^{2}+c_{1} \lambda+c_{0}$. The expressions of $c_{i}$ are rather lengthy and were omitted for brevity. The characteristic polynomial has two similar negative eigenvalues $\left(\lambda_{1}=\lambda_{2}=-m_{I}-\right.$ $\varpi)$. According to the Routh-Hurwitz stability criterion, the zeros of $H(\lambda)$ have $\operatorname{Re}(\lambda)<0$ if $c_{3}>0, c_{3} c_{2}-c_{1}>0, c_{1}\left(c_{3} c_{2}-c_{1}\right)-c_{3}^{2} c_{0}>$ 0 and $c_{0}>0$. Therefore, we can numerically estimate the critical transmissibility of disease through analyzing above four inequalities when the values of parameters are given.

\section{References}

Baek, H., 2009. Species extinction and permanence of an impulsively controlled twoprey one-predator system with seasonal effects. BioSystems 98, 7-18.

Bairagi, N., Roy, P.K., Chattopadhyay, J., 2007. Role of infection on the stability of a predator-prey system with several response functions-a comparative study. J. Theor. Biol. 248, 10-25.

Bauch, C.T., 2005. The spread of infectious diseases in spatially structured populations: an invasory pair approximation. Math. Biosci. 198, 217-237.

Bhattacharyya, R., Mukhopadhyay, B., 2010. On an eco-epidemiological model with prey harvesting and predator switching: local and global perspectives. Nonlinear Anal. 11, 3824-3833.

Bonsall, M.B., Hassell, M.P., 2000. The effects of metapopulation structure on indirect interactions in host-parasitoid assemblages. Proc. R. Soc. Lond. B 267, 2207-2212.

Boots, M., Sasaki, A., 1999. 'Small worlds' and the evolution of virulence: infection occurs locally and at a distance. Proc. R. Soc. Lond. B 266, 1933-1938.

Chattopadhyay, J., Bariagi, N., 2001. Pelicans at risk in Salton Sea-an ecoepidemiological study. Ecol. Model. 136, 103-112.

Chattopadhyay, J., Sarkar, R.R., Ghosal, G., 2002. Removal of infected prey prevent limit cycle oscillations in an infected prey-predator system-a mathematical study. Ecol. Model. 156, 113-121.

Dawkins, R., 1982. The Extended Phenotype: The Long Reach of the Gene. Oxford University Press, Oxford.

Delgado, M., Molina-Becerra, M., Suárez, A., 2006. Analysis of an age-structured predator-prey model with disease in the prey. Nonlinear Anal. 7, 853-871.

Fortin, M.J., Dale, M.R.T., 2005. Spatial Analysis: A Guide for Ecologists. Cambridge University Press, Cambridge.

Greenman, J., Hoyle, A., 2010. Pathogen exclusion from eco-epidemiological systems. Am. Nat. 176, 149-158.

Hall, S.R., Duffy, M.A., Cáceres, C.E., 2005. Selective predation and productivity jointly drive complex behavior in host-parasite systems. Am. Nat. 165, 70-81.

Hatcher, M.J., Dick, J.T.A., Dunn, A.M., 2006. How parasites affect interactions between competitors and predators. Ecol. Lett. 9, 1253-1271.

Hatcher, M.J., Dick, J.T.A., Dunn, A.M., 2008. A keystone effect for parasites in intraguild predation? Biol. Lett. 4, 534-537.

Hethcote, H.W., Wang, W., Han, L., Ma, Z., 2004. A predator-prey model with infected prey. Theor. Popul. Biol. 66, 259-268.

Hiebeler, D.E., 2005. A cellular automaton SIS epidemiological model with spatially clustered recoveries. Lect. Notes Comput. Sci. 3515, 360-367.
Holt, R.D., Roy, M., 2007. Predation can increase the prevalence of infectious disease Am. Nat. 169, 690-699.

Hudson, P.J., Dobson, A.P., Newborn, D., 1992. Do parasites make prey vulnerable to predation? Red grouse and parasites. J. Anim. Ecol. 61, 681-692.

Hui, C., McGeoch, M.A., Warren, M., 2006. A spatially explicit approach to estimating species occupancy and spatial correlation. J. Anim. Ecol. 75, 140-147.

Hui, C., McGeoch, M.A., 2007. Spatial patterns of prisoner's dilemma game in metapopulations. Bull. Math. Biol. 69, 659-676.

Hui, C., Veldtman, R., McGeoch, M.A., 2010. Measures, perceptions and scaling patterns of aggregated species distributions. Ecography 33, 95-102.

Iwasa, Y., 2000. Lattice models and pair approximation in ecology. In: Dieckmann, U., Law, R., Metz, J. (Eds.), The Geometry of Ecological Interactions: Simplifying Spatial Complexity. Cambridge University Press, Cambridge.

Joly, D.O., Messier, F., 2004. The distribution of Echinococcus granulosus in moose: evidence for parasite-induced vulnerability to predation by wolves? Oecologia 140, 586-590.

Keeling, M.J., Rand, D.A., 1996. Spatial correlations and local fluctuations in host-parasite ecologies. In: Glendinning, P. (Ed.), From Finite to Infinite Dimensional Systems. Kluwer, Amsterdam.

Kihara, K., Mori, K., Suzuki, S., Hosoda, K., Yamada, A., Matsuyama, S.I., Kashiwagi, A. Yomo, T., 2011. Probabilistic transition from unstable predator-prey interaction to stable coexistence of Dictyostelium discoideum and Escherichia coli. BioSystems $103,342-347$.

Lafferty, K.D., Allesina, S., Arim, M., Briggs, C.J., et al., 2008. Parasites in food webs: the ultimate missing links. Ecol. Lett. 11, 533-546.

Mata-Machuca, J.L., Martínez-Guerra, R., Aguilar-López, R., 2010. Monitoring in a predator-prey systems via a class of high order observer design. BioSystems 100, 65-69.

Nie, L., Teng, Z., Hu, L., Peng, J., 2009. The dynamics of a Lotka-Volterra predator-prey model with state dependent impulsive harvest for predator. BioSystems 98 67-72.

O'Keefe, K.J., 2005. The evolution of virulence in pathogens with frequencydependent transmission. J. Theor. Biol. 233, 55-64.

Okuyama, T., 2008. Intraguild predation with spatially structured interactions. Basic Appl. Ecol. 9, 135-144.

Ostfeld, R.S., Holt, R.D., 2004. Are predators good for your health? Evaluating evidence for top-down regulation of zoonotic disease reservoirs. Frontiers Ecol. Environ. 2, 13-20.

Packer, C., Holt, R.D.,Hudson, P.., Lafferty, K.D., Dobson, A.P., 2003. Keeping the herds healthy and alert: implications of predator control for infectious disease. Ecol. Lett. 6, 797-802.

Raffel, T.R., Martin, L.B., Rohr, J.R., 2008. Parasites as predators: unifying natural enemy ecology. Trends Ecol. Evol. 23, 610-619.

Raffel, T.R., 2010. Parasitism in a community context: trait-mediated interactions with competition and predation. Ecology 91, 1900-1907.

Rousset, F., 1996. Inference of parasite-induced host mortality from distributions of parasite loads. Ecology 77, 2203-2211.

Roy, M., Holt, R.D., 2008. Effects of predation on host-pathogen dynamics in SIR models. Theor. Popul. Biol. 73, 319-331.

Satō, K., Matsuda, H., Sasaki, A., 1994. Pathogen invasion and host extinction in lattice structured populations. J. Math. Biol. 32, 251-268.

Shaw, D.J., Grenfell, B.T., Dobson, A.P., 1998. Patterns of macroparasite aggregation in wildlife host populations. Parasitology 117, 597-610.

Slone, D.H., 2011. Increasing accuracy of dispersal kernels in grid-based population models. Ecol. Model. 222, 573-579.

Su, M., Hui, C., Zhang, Y.Y., Li, Z.Z., 2009a. How does the spatial structure of habitat loss affect the eco-epidemic dynamics? Ecol. Model. 220, 51-59.

Su, M., Li, W.L., Li, Z.Z., Zhang, F.P., Hui, C., 2009b. The effect of landscape heterogeneity on host-parasite dynamics. Ecol. Res. 24, 889-896.

Sun, G.Q., Jin, Z., Liu, Q.X., Li, B.L., 2010. Rich dynamics in a predator-prey model with both noise and periodic force. BioSystems 100, 14-22.

Tilman, D., Kareiva, P., 1997. Spatial Ecology: The Role of Space in Population Dynamics and Interspecific Interactions. Princeton University Press, Princeton.

Webb, S.D., Keeling, M.J., Boots, M., 2007a. Host-parasite interactions between the local and the mean-field: how and when does spatial population structure matter? J. Theor. Biol. 249, 140-152.

Webb, S.D., Keeling, M.J., Boots, M., 2007b. Spatially extended host-parasite interactions: the role of recovery and immunity. Theor. Popul. Biol. 71, 251-266.

Williams, P.D., 2008. Unhealthy herds: some epidemiological consequences of host heterogeneity in predator-host-parasite systems. J. Theor. Biol. 253, 500-507.

Woodcock, B.A., Watt, A.D., Leather, S.R., 2002. Aggregation, habitat quality and coexistence: a case study on carrion fly communities in slug cadavers. J. Anim. Ecol. $71,131-140$ 